\title{
Queueing Systems With Multiple Servers Under Differentiated Working Vacations
}

\author{
Vyshna Unni $^{1}$, K. Julia Rose Mary ${ }^{2}$ \\ Department of mathematics ${ }^{1,2}$ \\ Nirmala College for Women \\ Red fields, Coimbatore - 641 018, India \\ Email:vyshnaunni@gmail.com ${ }^{1}$, juliakulandaisamy@gmail.com ${ }^{2}$
}

\begin{abstract}
The implementation of server vacations in fundamental queueing systems had opened a broad platform for many of the upcoming researchers to develop rewarding theories for the society. The impacts of such inventions in the scientific field provoked many of them to extend the vacation strategies in various directions like single vacation, multiple vacations, working vacations and so on. Recently a new scheme named differentiated vacations related to the vacation periods in a multi vacations queueing system was emerged. In this paper we introduced the differentiated vacations policy into multi servers queueing systems with working vacations. That is in the proposed queueing system more than one server is available, each of the servers can go for two types of vacations with different durations and at the time of vacations they will not terminate their respective duty completely. We modeled the system and provided a detailed interpretation about the steady state probabilities of various states. We also derived the formulas for calculating some performance measures of the recommended queueing model and discussed the nature of the average waiting time generated in the system with different choices for various values of the parameters. We also analyzed the relation between the durations of vacations and number of servers in the system.
\end{abstract}

Keywords-Multi server queueing systems; Vacation queueing systems; Differentiated vacations; Average waiting time; Working vacations.

\section{INTRODUCTION}

Queueing systems with server vacations are the area of interest to many researchers in recent years due to its immense applications in our daily life situations. The field of queueing systems with server vacations plays a vital role in research works because it reduces the working pressure of the employees and the provocation of the customers in the system. A vacation queueing system is characterized by the fact that the servers may become unavailable for a certain period of time from the primary service center due to some circumstances. The time that the server is away from the system is termed as a vacation in the system. There are many reasons for a server to take a break from the system, which includes power saving mode, insufficient work load in human behavior, preventive maintenance period in a productive system and so on. The idea of introducing the vacation scheme into the queueing system was first done by Levy and Yenchiali [8] in 1975. An exhaustive study of vacation queueing system can be seen in the survey of Doshi $(1986,1990)[4,5]$ and also in the book of Takagi(1991)[13]. Many researchers like Tian and Zhang (2006) [14] have attracted widely into this research area and provided fruitful theories related to the concept.

There are different types of vacation policies such as single vacation, multiple vacations and working vacations which are introduced in queueing systems according to many real life situations. In single vacation policy the server takes a vacation of some random period and returns to normal mode even if there is no customers are waiting in the system. But in multiple vacations queueing systems the server takes vacations until he finds a waiting customer. Servi and Finn (2002)[10] introduced the third type of vacation scheme in which the server works at different rate rather than completely stopping the service during a vacation. They studied about wave length division multiplexing optical access network by using $M / M / 1$ queueing systems with multiple vacations. With the aid of Servi and Finn's concept many researchers developed queueing models with working vacations like Baba (2005)[1] studied about $G I / M / 1$ queue with working vacations by using matrix analytical method. $\mathrm{Wu}$ and Takagi (2006) [16] generalized the model given by Servi and Finn to $M / G / 1$ queue with general working vacations. Baniket al. (2007) [3] discussed about $G I / M / 1 / N$ queue with working vacations. A stochastic decomposition result in $M / M / 1$ queue with working vacations presented in the paper of Liu et al. (2007)[9]. Tian et al. (2008)[15] considered the discrete time Geo/Geo/1 queue with multiple working vacations. The working vacation policies were also extending into queueing models having batch arrival queues. Xu et al. (2009) [17] studied a batch arrival $M^{X} / M / 1$ queue with single working vacation;whereas Baba (2012)[2] studied the respective model with multiple working vacations policies.

Major classes of research works with multiple vacations strategy were developed by assuming that the successive vacations occur in the system will follow the same distribution with same mean. Recently a new concept named differentiated vacations was introduced to differentiate the successive vacations taken by the servers in the system. Some basic structures of differentiated vacations can be seen in the research works of Fiems et al. (2004)[6] and Vishenvsky et al.(2011)[12]. But a detailed interpretation on differentiated vacation was given by Oliver C Ibe and Olubukola A Isijola in 2014 [7]. They discussed deeply about a $M / M / 1$ queueing systems where servers have the opportunity to go for two types of vacations having different durations. They give the steady state analysis of the model and described about the 


\section{International Journal of Research in Advent Technology, Vol.7, No.4, April 2019 \\ E-ISSN: 2321-9637 \\ Available online at $w w w . i j r a t . o r g$}

performance measures of the queueing system. A transient state analysis of this model was presented by Vijayashree and Janani in 2018 [11]. They provided the explicit expressions for the time depended system size probabilities in terms of modified Bessel function of second kind.

In this paper we discussed about a multiple server queueing system with differentiated working vacations. The proposed model is designed with $C$ servers and each of the servers in the system has the option to take two kinds of vacations. But during the period of each type of vacations the servers will work at a different rate instead of completely stopping the service. The servers in the system are allowed to go for the first type of vacation only after completing a non zero busy period of each of the servers and if there are no customers are waiting in any of the $C$ queues when they returned from the first type of vacation then only the servers have the permission to take the second type of vacation. But there are separate timings and fixed lengths for each type of vacations that we assigned for the servers in the system.

The proposed queueing system can be widely introduced to model many of our real life situations to avoid the stress of the employees. Also this model supports the sincere servers of the system through the working vacation policies. So the customers arrived in to the system will be satisfied soon due to a very less waiting time generated in the system.

The rest of the paper is organized as follows. Section 2 described about the model under consideration. Section 3 discussed about the steady state probabilities and performance measures of the proposed model. Section 4 presented some numerical examples with graphical interpretation and the observations obtained about the model are concluded in section 5 .

\section{SYSTEM MODEL WITH DIFFERENTIATED VECATIONS}

In this section we examine about a multiple server queueing model with differentiated vacations. For that we consider an $M / M / C$ queueing systems where customers arrive according to Poisson process with rate $\lambda$ and the time to serve is exponentially distributed with mean $1 / \mu$, where $\mu>\lambda$. The servers of the proposed model are allowed to go for two types of vacations with disparate durations, where type 1 of vacation will be started by the servers when they become idle. But before entering to this vacation it should be ensured that all of the $C$ servers have treated at least one customer in the system. The length of this vacation is supposed to be exponentially distributed with mean $1 / \gamma_{1}$. When the servers returned from this vacation, if no customers are waiting in any of the $C$ for service then the servers are allowed to go for another type of vacations called type 2 . These types of vacations can be repeated as long as the system is empty. The duration of this vacation is also assumed to be distributed exponentially with mean $1 / \gamma_{2}$. The remarkable characteristic of our recommended model is that the $C$ servers of the system still process their jobs in the allowed periods of both the vacations at different service rates. That is at the time of type 1 vacation, each of the $C$ servers will perform their respective duty with the fixed service rate say $\mu_{1}$ and the case is similar for type 2 vacation also but we considered the service rate as $\mu_{2}$. From the nature of the vacations that we offered, it is obvious that $\mu_{2}>\mu_{1}$ and $\mu>$ $\mu_{1}, \mu_{2}$. Let us denote the state of the system as $(n, m)$, where $n$ is the number of customers in the system and $m$ stands for the nature of the vacation in which the servers are currently occupied. That is if $m=1$, then all the servers are in type 1 vacation, $m=2$, then all the servers are in type 2 vacation and finally if $m=0$, then all them are in active mode. Thus the system can be modeled by a continuous time markov chain whose state transition diagram is given in Fig. 1.

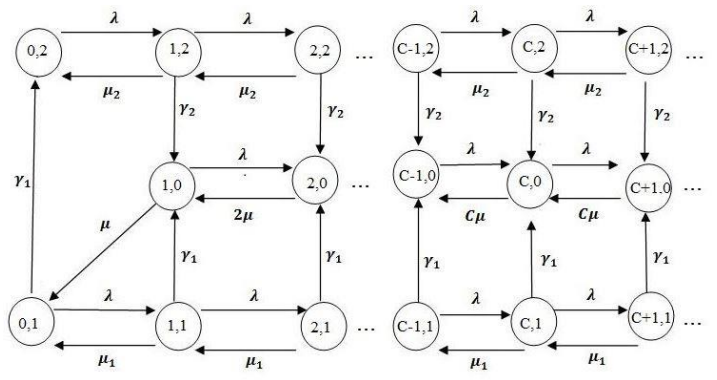

Fig. 1. State transition diagram

\section{STEADY STATE ANALYSIS}

Let $P_{n, m}$ denote the steady state probability that the system is in the state $(n, m)$. Now the global balance equations at state $(0,1)$ and $(0,2)$ are given by

$$
\begin{aligned}
& \quad\left(\lambda+\gamma_{1}\right) P_{0,1}=\mu P_{1,0}+\mu_{1} P_{1,1} \\
& \text { and } \\
& \lambda P_{0,2}=\gamma_{1} P_{0,1}+\mu_{2} P_{1,2} .
\end{aligned}
$$

By analyzing the local balances at states $(n+1,1)$ and $(n+1,2)$ for $n=0,1,2 \ldots$, we obtain the following equations,

$\lambda P_{n, 1}=\left(\mu_{1}+\gamma_{1}\right) P_{n+1,1}$
and
$\lambda P_{n, 2}=\left(\mu_{2}+\gamma_{2}\right) P_{n+1,2}$

By solving equations (3) and (4) recursively we obtain for $n=1,2,3 \ldots$

$$
\begin{array}{r}
P_{n, 1}=\left(\frac{\lambda}{\mu_{1}+\gamma_{1}}\right)^{n} P_{0,1} \\
\text { and } P_{n, 2}=\left(\frac{\lambda}{\mu_{2}+\gamma_{2}}\right)^{n} P_{0,2}
\end{array}
$$

Let $\beta_{1}=\frac{\lambda}{\mu_{1}+\gamma_{1}}$ and $\beta_{2}=\frac{\lambda}{\mu_{2}+\gamma_{2}}$. With these notations we can write

$$
P_{n, 1}=\beta_{1}^{n} P_{0,1}
$$

and

$$
P_{n, 2}=\beta_{2}{ }^{n} P_{0,2}
$$

In order to maintain our system to be perfect, we assume that $\beta_{1}$ and $\beta_{2}$ should be less than one. Thus from Eq. (5) we have,

$P_{1,1}=\left(\frac{\lambda}{\mu_{1}+\gamma_{1}}\right) P_{0,1}$

Substitute the above equations in Eq. (1) gives,

$$
\left(\lambda+\gamma_{1}\right) P_{0,1}=\mu P_{1,0}+\frac{\mu_{1 \lambda}}{\mu_{1}+\gamma_{1}} P_{0,1} .
$$

Now by solving Eq. (10) for $P_{0,1}$ gives

$$
P_{0,1}=\alpha_{1} P_{1,0},
$$


where $\alpha_{1}=\frac{\mu\left(\mu_{1}+\gamma_{1}\right)}{\gamma_{1}\left(\lambda+\mu_{1}+\gamma_{1}\right)}$. Similarly from Eq. (6) we can write,

$$
P_{1,2}=\frac{\lambda}{\mu_{2}+\gamma_{2}} P_{0,1}
$$

By substituting equations (12) in (2) and by using Eq. (11) we can derive that

$$
P_{0,2}=\alpha_{2} P_{1,0}
$$

where $\alpha_{2}=\frac{\mu\left(\mu_{1}+\gamma_{1}\right)\left(\mu_{2}+\gamma_{2}\right)}{\gamma_{2} \lambda\left(\lambda+\mu_{1}+\gamma_{1}\right)}$. By using the expressions for $P_{0,1}$ and $P_{0,2}$ given in equations (11) and (13), we can write for $n=1,2,3 \ldots$

$$
P_{n, 1}=\alpha_{1} \beta_{1}^{n} P_{1,0}
$$$$
P_{n, 2}=\alpha_{2} \beta_{2}^{n} P_{1,0}
$$

In order to obt probabilities of active mode, we go for the local balances at state $(n+1,0)$ where $n=1,2,3 \ldots$ then we obtain the following equations.

$$
\begin{aligned}
& \lambda P_{n, 0}+\lambda P_{n, 1}+\lambda P_{n, 2}=n \mu P_{n+1,0} \\
& \text { for } n=1,2, \ldots C-1 \text { and } \\
& \lambda P_{n, 0}+\lambda P_{n, 1}+\lambda P_{n, 2}=C \mu P_{n+1,0}
\end{aligned}
$$

for $n=C, C+1, C+2, \ldots$

By taking $\rho=\frac{\lambda}{\mu}$, we obtain

$P_{n .0}= \begin{cases}\frac{\rho}{n}\left(P_{n-1,0}+P_{n-1,1}+P_{n-1,2}\right), & n<C \\ \frac{\rho}{C}\left(P_{n-1,0}+P_{n-1,1}+P_{n-1,2}\right), & n \geq C\end{cases}$

Now by considering each case of Eq. (18) separately and by solving them recursively we obtain,

$P_{n .0}=\left\{\begin{array}{l}\left(\frac{\rho^{n-1}}{n !}+\sum_{i=0}^{n-2} \frac{\rho^{n-(i+1)}(i+1) !}{n !}\left[\alpha_{1} \beta_{1}^{i+1}+\alpha_{2} \beta_{2}^{i+1}\right]\right) P_{1,0}, n<C \\ \left(\left(\frac{\rho}{C}\right)^{n-C} P_{C, 0}+\sum_{i=1}^{n-C}\left(\frac{\rho}{C}\right)^{i}\left[\alpha_{1} \beta_{1}^{n-i}+\alpha_{2} \beta_{2}^{n-i}\right]\right) P_{1,0}, n \geq C .\end{array}\right.$

Now it is enough to find out the value of $P_{1,0}$ to get all the steady state probabilities at different states. For that we consider total probability concept, which leads to $\sum_{n=1}^{\infty} P_{n, 0}+\sum_{n=0}^{\infty} P_{n, 1}+\sum_{n=0}^{\infty} P_{n, 2}=1$.

That is,

$$
\begin{gathered}
\left\{\sum_{n=1}^{C} P_{n, 0}+\frac{\rho}{C-\rho}\left\{P_{C, 0}+\frac{\alpha_{1} \beta_{1}^{C}}{1-\beta_{1}}+\frac{\alpha_{2} \beta_{2}^{C}}{1-\beta_{2}}\right\}+\frac{\alpha_{1}}{1-\beta_{1}}\right. \\
\left.+\frac{\alpha_{2}}{1-\beta_{2}}\right\} P_{1,0}=1
\end{gathered}
$$

Thus we have

$$
\begin{aligned}
P_{1,0}= & \left(\sum_{n=1}^{C} P_{n, 0}+\frac{\rho}{C-\rho}\left\{P_{C, 0}+\frac{\alpha_{1} \beta_{1}^{C}}{1-\beta_{1}}+\frac{\alpha_{2} \beta_{2}^{C}}{1-\beta_{2}}\right\}+\right. \\
& \left.\frac{\alpha_{1}}{1-\beta_{1}}+\frac{\alpha_{2}}{1-\beta_{2}}\right)^{-1}
\end{aligned}
$$

The average queue length, $E(m)$ of a queueing system with $C$ - servers is given by

$E(m)=\sum_{n=C}^{\infty}(n-C) P_{n}$. Thus in the proposed model of working vacation queueing system, the average queue length is given by

$$
\begin{gathered}
E(m)=\sum_{n=C}^{\infty}(n-C) P_{n, 0}+\sum_{n=C}^{\infty}(n-C) P_{n, 1} \\
+\sum_{n=C}^{\infty}(n-C) P_{n, 2}
\end{gathered}
$$

Hence by substituting the respective probabilities we can derive that

$$
\begin{aligned}
E(m)= & \left\{\frac{C \rho}{(C-\rho)^{2}} P_{C, 0}+\frac{\alpha_{1} \beta_{1}^{C+1}}{\left(1-\beta_{1}\right)^{2}}+\frac{\alpha_{2} \beta_{2}^{C+1}}{\left(1-\beta_{2}\right)^{2}}+\right. \\
& \frac{\alpha_{1} \rho}{(C-\rho)\left(1-\beta_{1}\right)}\left(\frac{C \beta_{1}^{C}-(C-1) \beta_{1}^{C+1}}{1-\beta_{1}}+\frac{C \beta_{1}^{C}(1-C+\rho}{C-\rho}\right)+ \\
& \frac{\alpha_{2} \rho}{(C-\rho)\left(1-\beta_{2}\right)}\left(\frac{C \beta_{2}^{C}-(C-1) \beta_{2}^{C+1}}{1-\beta_{2}}+\right. \\
& \left.\left.\frac{C \beta_{2}^{C}(1-C+\rho}{C-\rho}\right)\right\} P_{1,0}
\end{aligned}
$$

Hence from Little's formula the average waiting time in the system $E(v)$ is found by using

$$
E(v)=\frac{E(m)}{\lambda}+\frac{1}{\mu}
$$

\section{NUMERICAL EXAMPLES}

In this section we analyze the effect of parameters on performance measures for the model presented in section 2. Throughout the discussion we fixed the parameters $\mu, \mu_{1}$ and $\mu_{2}$ as $0.4,0.25$, and 0.3 respectively. Since we considered a multiple server queueingsystem we choose two values for the number of servers $(C)$, which are $C=$ 3 and $C=5$. We assume that the mean duration of type 1 vacation is larger than that of type 2 vacation, that is $\gamma_{2} \geq \gamma_{1}$, where type 1 vacation is taken after the completion of a busy period of non zero duration of each of the servers and type 2 vacation is taken if there are no customers are waiting in any of the Cqueues for service when the servers are returned from the type 1 vacation.

First let us fix the value of $\gamma_{2}$ as 0.1 . By varying the value of $\gamma_{1}$ as 0.06 and 0.01 respectively, we evaluated the average queue length $(E(m))$ and the averagewaiting time $(E(v))$ for each choice of $C$ at some selected values of $\rho$.The obtained values of $E(m)$ and $E(v)$ in each case are tabulated in table 1 . 


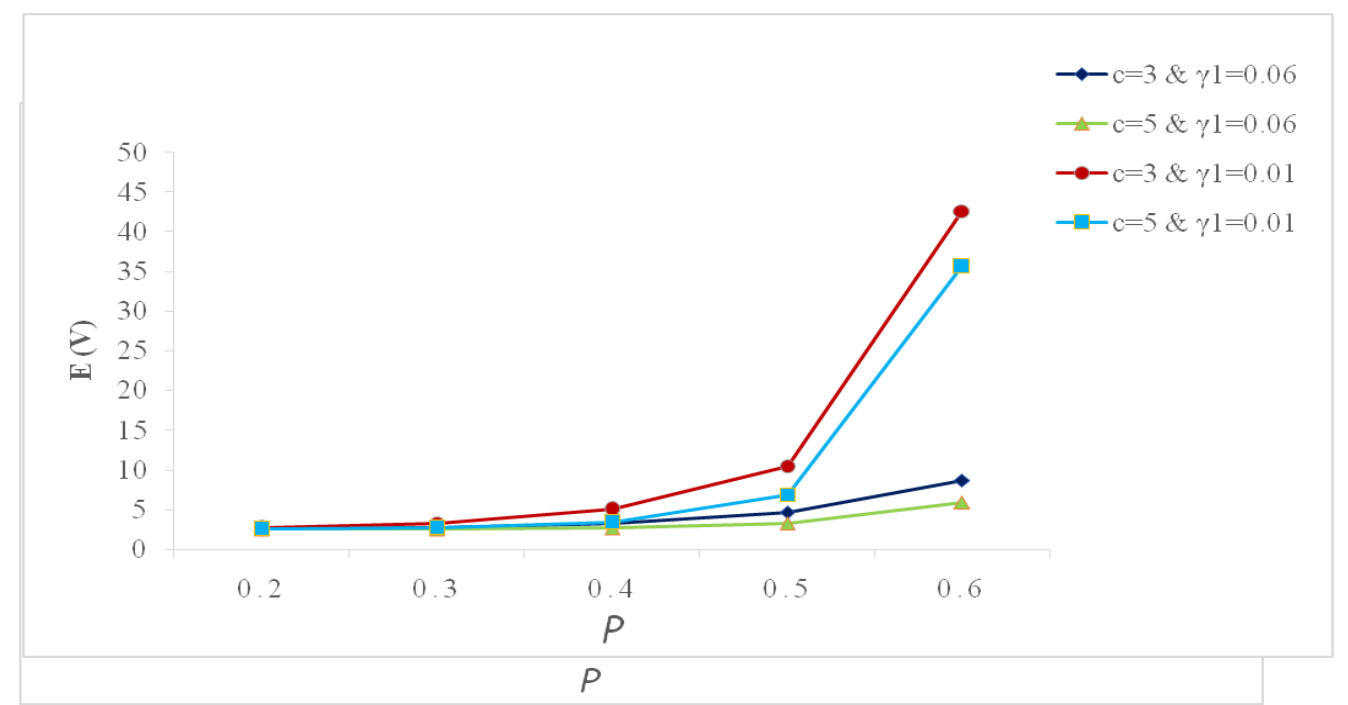

Fig. 3. Mean time in the system by varying $\rho$ for $\gamma_{2}=0.5$

Fig. 2. Mean time in the system by varying $\rho$ for $\gamma_{2}=0.1$

Table 1. $E(m)$ and $E(v)$ for $\gamma_{2}=0.1$

\begin{tabular}{|c|c|c|c|c|c|}
\hline$\gamma_{1}$ & $\rho$ & \multicolumn{2}{|c|}{$\boldsymbol{E}(\boldsymbol{m})$} & \multicolumn{2}{c|}{$\boldsymbol{E}(\boldsymbol{v})$} \\
\cline { 3 - 6 } & & $\boldsymbol{C = 3}$ & $\boldsymbol{C = 5}$ & $\boldsymbol{C}=\mathbf{3}$ & $\boldsymbol{C}=\mathbf{5}$ \\
\hline \multirow{4}{*}{0.06} & 0.2 & 0.0041 & 0.0001 & 2.5512 & 2.5023 \\
\cline { 2 - 6 } & 0.3 & 0.0263 & 0.0028 & 2.7198 & 2.5241 \\
\cline { 2 - 6 } & 0.4 & 0.1085 & 0.0224 & 3.1785 & 2.6400 \\
\cline { 2 - 6 } & 0.5 & 0.3683 & 0.1250 & 4.3416 & 3.1251 \\
\cline { 2 - 6 } & 0.6 & 1.2325 & 0.6381 & 7.6356 & 5.1591 \\
\hline \multirow{4}{*}{0.01} & 0.2 & 0.0119 & 0.0009 & 2.6491 & 2.5121 \\
\cline { 2 - 6 } & 0.3 & 0.0826 & 0.0155 & 3.1891 & 2.6293 \\
\cline { 2 - 6 } & 0.4 & 0.3727 & 0.1269 & 4.8298 & 3.2933 \\
\cline { 2 - 6 } & 0.5 & 1.5166 & 0.8296 & 10.0833 & 6.6481 \\
\cline { 2 - 6 } & 0.6 & 9.4068 & 7.7521 & 41.6952 & 34.8006 \\
\hline
\end{tabular}

From the table we can perceive that the average queue length in the system becomes larger if we enlarge the mean duration of the type 1 vacation. More precisely in a 3 - server queueing system if $\gamma_{1}$ takes the value 0.01 , then the increment of average waiting time in the system processes quickly when compared to the average waiting time of the respective queueing system with $\gamma_{1}=0.06$. We reach in a similar observation if we consider a 5server queueing system with the same variations for the values of the parameter $\gamma_{1}$. But a major conclusion obtained for us that the average waiting time is relatively higher in a 3 -server queueing system even if we choose $\gamma_{1}$ to be a larger value.

Fig.2 shows the graphical interpretation of the above description. From the graph we obtained an overview about the nature of average waiting time generated in both 3 and 5 servers queueing systems with the selected values of the parameters. If we observe the graph corresponding to $C=3$ and $\gamma_{1}=0.01$, we can notice that comparatively higher than the average waiting time in all of the remaining cases. Also we can see that if we choose $\gamma_{1}$ to be a smaller value then $E(v)$ is tending to approach its peak value as $\rho$ approaches to its boundary value 1 .

Next let us fix $\gamma_{2}=0.5$ and vary $\gamma_{1}$ as 0.06 and 0.01 respectively. Proceeded for $E(m)$ and $E(v)$ as in the previous case and the resulted values of them at some selected values of $\rho$ and each value of $C$ are given in table 2. The graphical representation of this case is also drawn in Fig. 3 and the graphs show that $E(v)$ behaves as in the first case. In other words in this case also the 5servers queueing system with $\gamma_{1}=0.06$ will provide the minimum average waiting time in the system.

From each of the considered cases it can be noted that a small change made in the value of $\gamma_{1}$ affect the average waiting time of the system noticeably than a change made in the value of $\gamma_{2}$.

Table 2. $E(m)$ and $E(v)$ for $\gamma_{2}=0.5$

\begin{tabular}{|c|c|c|c|c|c|}
\hline$\gamma_{\mathbf{1}}$ & $\boldsymbol{\rho}$ & \multicolumn{2}{|c|}{$\boldsymbol{E}(\boldsymbol{m})$} & \multicolumn{2}{c|}{$\boldsymbol{E}(\boldsymbol{v})$} \\
\cline { 3 - 6 } & & $\boldsymbol{C = 3}$ & $\boldsymbol{C = 5}$ & $\boldsymbol{C = 3}$ & $\boldsymbol{C = 5}$ \\
\hline \multirow{4}{*}{0.06} & 0.2 & 0.0038 & 0.0002 & 2.5483 & 2.5027 \\
\cline { 2 - 6 } & 0.3 & 0.0281 & 0.0036 & 2.7347 & 2.5302 \\
\cline { 2 - 6 } & 0.4 & 0.1242 & 0.0288 & 3.2762 & 2.6805 \\
\cline { 2 - 6 } & 0.5 & 0.4392 & 0.1621 & 4.6960 & 3.3107 \\
\cline { 2 - 6 } & 0.6 & 1.4933 & 0.8138 & 8.7221 & 5.8909 \\
\hline \multirow{4}{*}{0.01} & 0.2 & 0.0137 & 0.0011 & 2.6714 & 2.5145 \\
\cline { 2 - 6 } & 0.3 & 0.0917 & 0.0176 & 3.2649 & 2.6469 \\
\cline { 2 - 6 } & 0.4 & 0.4030 & 0.1390 & 5.0192 & 3.3692 \\
\cline { 2 - 6 } & 0.5 & 1.5997 & 0.8807 & 10.4988 & 6.9037 \\
\cline { 2 - 6 } & 0.6 & 9.6180 & 7.9411 & 42.57 & 35.588 \\
\hline
\end{tabular}




\section{International Journal of Research in Advent Technology, Vol.7, No.4, April 2019 E-ISSN: 2321-9637 \\ Available online at www.ijrat.org}

\section{CONCLUSION}

In this paper we proposed a multiple servers queueing system with differentiated working vacations. So the servers of the suggested model will never terminate their jobs completely during the allowed time of vacations. Instead of that they will processes their respective duties at different rates depending upon the nature of the vacations in which they are currently occupied. We derived the formulas for calculating the steady state probabilities, average queue length and average waiting time of the system. Further we studied about the behavior of the average waiting time in the system with various choices for the durations of vacations. The mean time that a customer spends in the system is more sensitive to the mean duration of the first type of vacation. But if we increase the number of servers in the system, we can correspondingly increase the durations of vacations offered for the servers by balancing the average waiting time of the system within a favorable range.

The recommended model can be widely introduced in various streams of business industries. Obviously developing a strong bond between customers and servers is the major technique adopted by the business corporate for their successful movement. Due to the presence of vacation strategies in our requested model the servers get more time to relax and hence they will work sincerely for the customers when they are in active mode. This attitude of the servers will evidently attract the customers into the system. Hence the business organization gets more profit and run successfully.

\section{ACKNOWLEDGMENTS}

The authors would like to express their sincere thanks to associate editors and anonymous reviewers for their useful and rigorous comments which have improved the quality of the manuscript.

\section{REFERENCES}

[1] Y. Baba. "Analysis of a G1/M/1 queue with multiple working vacations". Operations Research Letters, volume 33, pages 201-209, 2005.

[2] Y. Baba. "The $\mathrm{M}^{\mathrm{X}} / \mathrm{M} / 1$ queue with multiple working vacation". American Journal of Operations Research, volume 2, pages 217-224, 2012.

[3] A. D. Banik, U. C. Gupta and S. S. Pathak. "On the $\mathrm{GI} / \mathrm{M} / 1 / \mathrm{N}$ queue with multiple working vacations-analytic analysis and computation". Applied Mathematical Modelling, volume 31, pages 1701-1710, 2007.

[4] B. T. Doshi. "Queueing systems with vacations- a survey". Queueing systems: Theory and Applications, volume 1, pages 29-66, 1986.

[5] B. T. Doshi. "Single server queues with vacations". Stochastic Analysis of Computer and Communication Systems, pages 217-265, 1990.
[6] D. Fiems, J. Walraevens and H. Bruneel. " The discrete-time gated vacation queue revisited". AEU-International Journal of Electronics and Communications, volume 58, pages 136- 141, 2004.

[7] Oliver C Ibe and Olubukola A Isijola. “ $M / M / 1$ multiple Vacation Queueing systems with Differentiated vacations". Modelling and Simulation in Engineering, 2014.

[8] Y. Levy and U. Yechiali. " Utilization of idle time in an M/G/1 queueing system”. Management Science, volume 22, pages 202-211, 1975.

[9] W. Liu, X. Xu and N. Tian. "Stochastic decompositions in the M/M/1 queue with working vacations". Operations Research Letters, volume 35, pages 595-600, 2007.

[10] L. D. Servi and S. G. Finn. "M/M/1 queue with working vacations (M/M/1/WV)". Performance Evaluation, volume 50, pages 41-52, 2002.

[11] K. V. Vijayashree and B. Janani. "Transient analysis of an M/M/1 queueing system subject to diffrentiated vacations". Quality Technology and Quantitative Management, volume 15, pages 730-748, 2018.

[12] V. M. Vishnevsky, A. N. Dudin, O. V. Semenova and V. I. Klimenok. "Performance analysis of the BMAP/G/1 queue with gated servicing and adaptive vacations". Performance Evaluation, volume 68, pages 446-462, 2011.

[13] H. Takagi. "Queueing Analysis: A Foundation of Performance Analysis". vol. 1 of vacation and priority Systems, part 1, 1991.

[14] N. Tian and Z. G. Zhang. "Vacation Queueing Models: Theory and Applications". Springer, 2006.

[15] N. Tian, Z. Ma and M. Liu. "The discrete time Geom/Geom/1 queue with multiple working vacations". Applied Mathematical Modelling, volume 32, pages 2941-2953, 2008.

[16] D. $\mathrm{Wu}$ and $\mathrm{H}$. Takagi. "M/G/1 queue with multiple working vacations", Performance Evaluation, volume 63, pages 654-681, 2006.

[17] X. Xu, Z. Zhang and N. Tian. "Analysis for the $\mathrm{M}^{\mathrm{X}} / \mathrm{M} / 1$ working vacation queue". International Journal of information and Management Sciences, volume 20, pages 379-394, 2009. 